\title{
Modelling Spatial Whole-Part Relationships using an ISO-TC211 conformant approach
}

\author{
Alberto Belussi ${ }^{1}$, Mauro Negri ${ }^{2}$, Giuseppe Pelagatti ${ }^{2}$ \\ ${ }^{1}$ Dipartimento di Informatica, Università di Verona, Strada Le Grazie, 15, 37100, \\ Verona, Italy, tel:0039 +458027980 fax:0039 +2 8027982 \\ e-mail: alberto.belussi@univr.it \\ ${ }^{2}$ Dipartimento di Elettronica e Informazione, Politecnico di Milano, Via Ponzio,34/5, \\ 20133, Milano, Italy, tel:0039 +2 23993538 fax:0039 +2 23993411 \\ e-mail:negri@elet.polimi.it,pelagatt@elet.polimi.it,
}

Keywords: spatial whole part, ISO TC211, conceptual database design, modelling abstraction

\begin{abstract}
The important role of Spatial Whole-Part relationships in spatial database design is widely recognized and several researches have proposed specific spatial models to classify these relationships and their related topological constraints.

The recent ISO TC211 standards have defined a spatial data model and its use in spatial database design. However, the modelling of topological constraints of Spatial Whole-Part relationships requires additional complex and counterintuitive expressions specified by using a formal constraint language.

This paper shows the complexity of modelling Spatial Whole-Part relationships in ISO and proposes an approach for the definition of ISO conformant modelling abstractions which hide this kind of complexity to the database designer. This approach is applied to the definition of the modelling abstractions for Spatial Whole-Part relationships which cover all the Spatial Whole-Part relationships proposed in the literature.
\end{abstract}

\section{Introduction}

Geographical information systems deal with spatial data which are more complex and structured than those treated in traditional information systems and this has required an evolution of the available database technologies and of the database design approach.

The relational database technology has moved, through SQL99, towards the object oriented paradigm in order to capture complex data and algorithms in user defined data types and a specialization for spatial data has been standardized in SQL/MM.

Also the traditional conceptual modelling approaches have been extended to deal with the complexity and richness of spatial databases [10] in order to capture:

1. basic spatial data types, in terms of data structures (e.g., point, line, polygon) and operations (e.g., boundary, perimeter, area, etc.), for describing and manipulating the location and extent of the geometric objects embedded in a reference space of dimension 2 or 3 ;

2. spatial relationships (e.g., topological relationships like adjacency and containment) between geometric objects needed for querying spatial data;

3. spatial integrity constraints between spatial objects needed to maintain the integrity, and therefore the quality, of a spatial database.

Spatial relationships and constraints are very important in spatial databases $[10,11]$ because the spatial objects are embedded in a same geographical space.

A spatial Whole-Part relationship (called SWP relationship in the sequel) is a Whole-Part relationship in which whole and parts have a spatial extent and a topological constraint is defined between the spatial extent of the whole and the spatial extents of its parts that imposes a topological relationship among them. 
An example of SWP relationship is the relationship between an administrative county and its component administrative municipalities where the geometry of the county is partitioned in disjoint municipality areas.

The correct modelling of SWP relationships in conceptual database design is important because:

- it improves the understanding of the spatial content of the conceptual schema for both users and database designers, affecting the quality of data as perceived by the final user;

- it allows to provide mechanisms for their implementation (e.g., by means of topological structures, clustering and locking units) in the underlying database system.

The above two goals are obtained defining specific modelling abstractions for the SWP relationships, since this allows the designer to describe, and a user to recognize, common similar situations of the modelled world through a same modelling pattern. Notice that, the topological constraints of a SWP relationship could be expressed in terms of additional constraint formulas written using some general purpose constraint language attached to general Whole-Part relationships, however, these formulas are complex to write and hard to understand. Moreover, a topological constraint can be expressed using several different formulas and this is an obstacle for its classification in order to supply predefined implementations. Following this approach the result is therefore that the topological constraints are embedded in the application logic instead of the database.

The researches on SWP relationships have proposed several modelling abstraction, however all of them are not conformant to the ISO TC211 specifications, since they are based on different spatial data models.

ISO TC211 is defining the 19100 series of standards in order to standardize the management of the geographic information. Two of these standards refer to the conceptual specification of a spatial data model (ISO document 19107) [17] and to the rules to develop interoperable conceptual schemas for geographical databases (ISO document 19109) [18]; in the sequel they will be shortly called "Spatial Schema" and "Rules for Applications" respectively. The Spatial Schema has been also adopted by the OpenGeoSpatial Consortium (OGC) and restricted profiles for the implementation on relational database technology have been defined as ISO standards (ISO document 19125) [19] and as OGC specification [25]; some conformant implementations in database and GIS products are now available. The other standards of the 19100 series refer to other aspects (e.g., temporal data, grid models, etc.), which are not relevant to the scope of this paper.

A database designer can adopt the Spatial Schema and the Rules for Applications in the conceptual design of SWP relationships; in particular, the Contains association, which is defined in the Spatial Schema as a recursive association on the GM_Complex class, represents a structural mechanism for building complex topological constraints. However, the ISO approach does not define specific modelling abstractions and it requires the use of complex OCL (Object Constraint Language) expressions in spatial database design. This is due to the fact that the ISO standards emphasize only the intentional representation of the Spatial Schema, i.e., the standards define the abstract representation of the structure of the classes and the associations of the schema in order to support their implementation, but they do not force the behaviour of data. On the contrary in database design a schema is both an intentional and an extensional specification of the data: it defines data structures and how the instances must populate these structures when the database is implemented. The motivations of this paper are the following:

- to show the complexity of applying directly the ISO constructs and OCL formulas for the conceptual modelling of topological constraints of SWP relationships;

- to propose an approach for defining ISO conformant modelling abstractions which hide this kind of complexity;

- to apply this approach to the modelling of the SWP relationships, obtaining a minimal set of SWP modelling abstractions; these abstractions cover all the SWP relationships proposed in literature.

The paper is organized as follows: section 2 analyzes related works, section 3 describes the ISO hierarchy of spatial data types with their interrelationships, section 4 presents the ISO rules to 
define conceptual schemas using the spatial data types of section 3, section 5 investigates how the SWP relationships can be modelled directly in the ISO approach through OCL and the resulting problems and limitations. Finally, section 6 defines the ISO conformant abstractions for SWP relationships.

\section{Related works}

Whole-Part relationships (sometimes called aggregation, composition, meronymic relationship) has been extensively studied as a modelling concept in ontology, OO modelling and in databases $[2,6,15,20,21,24,31]$; also the Unified Modeling Language (UML) deals with these relationships through the notion of aggregation and composition [29]. However, different strategies have been adopted in order to define the characteristics of the Whole-Part relationship, resulting in overlapping meanings, contradictions and unclear semantics, as stated in [2].

A systematic classification of Whole-Part relationships properties is given in [2], where these relationships are defined in terms of mandatory primary characteristics (e.g., binary nature, asymmetry,...) and optional secondary characteristics (e.g, transitivity, shareability,...); the classification has not a formal justification, but it is driven by the experience and prior studies and therefore it is a pragmatic approach in order to implement these relationships in a possible UML revision. Although [2] recognizes the importance of having specific metamodeling constructs for the different kinds of Whole-Part relationships, it does not provide a taxonomy of the metamodeling constructs; it only proposes to extend UML through an abstract WP metatype (including all the primary properties) with the UML composition/aggregation metatypes (including some secondary property) as its specializations.

The researches on Whole-Part relationships do not consider the impact of spatial data on them and moreover not all the proposed types of Whole-Part relationships are relevant in database design [31].

Other researches have proposed conceptual models or design patterns for the design of spatial applications and databases $[1,3,4,7,8,9,12,13,14,22,23,26,27,28,30,32]$. These works define spatial data types and spatial relationships, but only some of them explicitly define modelling abstractions for SWP relationships [1,7,8,13,14,22,27,28] and only [27,28] explicitly analyze how SWP relationships apply the general classification of Whole-Part relationship properties of [2]. The above approaches consider different topological constraints between the whole and the parts or among the parts themselves and all the SWP relationships have not been defined as a result of an exhaustive analysis, but they are based on the experience [27], as the properties defined in [2].

The topological constraints of SWP relationships can be classified in the following three categories, based on the schema of [27]:

- containment constraint: the spatial extent of each part of a given whole must be included in the spatial extent of the whole (e.g., a building contained in the area of a building site);

- equality constraint: the spatial extent of the whole is equivalent to the geometric union of the spatial extent of its parts; it can admit overlapping among the spatial extents of the parts of a same whole or not (e.g., a road network composed by its roads);

- covering constraint: the spatial extent of the whole is contained in the geometric union of the spatial extent of its parts (e.g. a district area covered by the union of the broadcasting area of the transmitters of the district).

Table 1 shows how the SWP relationships proposed in the literature fall in the above categories. 


\begin{tabular}{|l|l|l|l|}
\hline \multicolumn{2}{|c|}{$\begin{array}{l}\text { Equality constraint } \\
\text { Overlapping admitted }\end{array}$} & Containment constraint & $\begin{array}{l}\text { Covering } \\
\text { constraint }\end{array}$ \\
\hline Spatial covering[1] & Spatial partition[1] & Spatial containment[7] & Spatial cover[14] \\
Spatial union[7] & Spatial subdivision[7] & Has_a[13] & Covering \\
Made_of[13] & Spatial aggregation[8] & Spatial_member_of[14] & structure[22] \\
Spatial_part_of[14] & Divided_into[13] & Containment & \\
Spatial aggregation [26] & Partition structure[22] & structure[22] & \\
Spatial derivation[27] & & Spatial inclusion[27,28] & \\
Spatial equal[28] & & & \\
\hline
\end{tabular}

Table 1. The classification of the SWP relationships.

\section{The ISO Spatial Schema}

The Spatial Schema deals with geometric and topological characteristics of spatial objects up to 3 topological dimensions embedded in coordinate spaces of up to 3 dimensions. It considers vector geometry and topological structures (by means of topological complexes), but the latter are not considered in this paper, except for those "topological aspects" included in the geometric model (topological complexes have been introduced in the standard mainly in order to reduce the overhead of the geometric computational algorithms). The standard aims to provide an object-oriented representation of the adopted mathematical interpretation of space; the motivations behind this object-oriented approach are:

- to supply the conceptual classes describing geometric properties that can be used in conceptual design of spatial databases and

- to provide the specification for the implementation of the Spatial Schema in underlying GIS systems; it defines a set of spatial data types, their attributes, operators and interrelationships using the UML notation.

Figure 1 shows the main classes of the Spatial Schema in which the overall set of types is organized in a hierarchy in order to share common characteristics.

The primitives (GM_primitive) are the geometric building blocks of the Spatial Schema and are used to build aggregates (GM_aggregate) and complex objects (GM_complex). The primitives are open and therefore they do not contain their boundaries (e.g., a curve does not contain its start/end points). The constructor method of a primitive allows the composition of the primitive as a connected set of component parts (e.g., a curve is composed of curve segments) in order to apply different interpolation methods for the acquisition of the geometry of each component.

Topological relationships among primitives are defined using the point-set representation of the objects. 


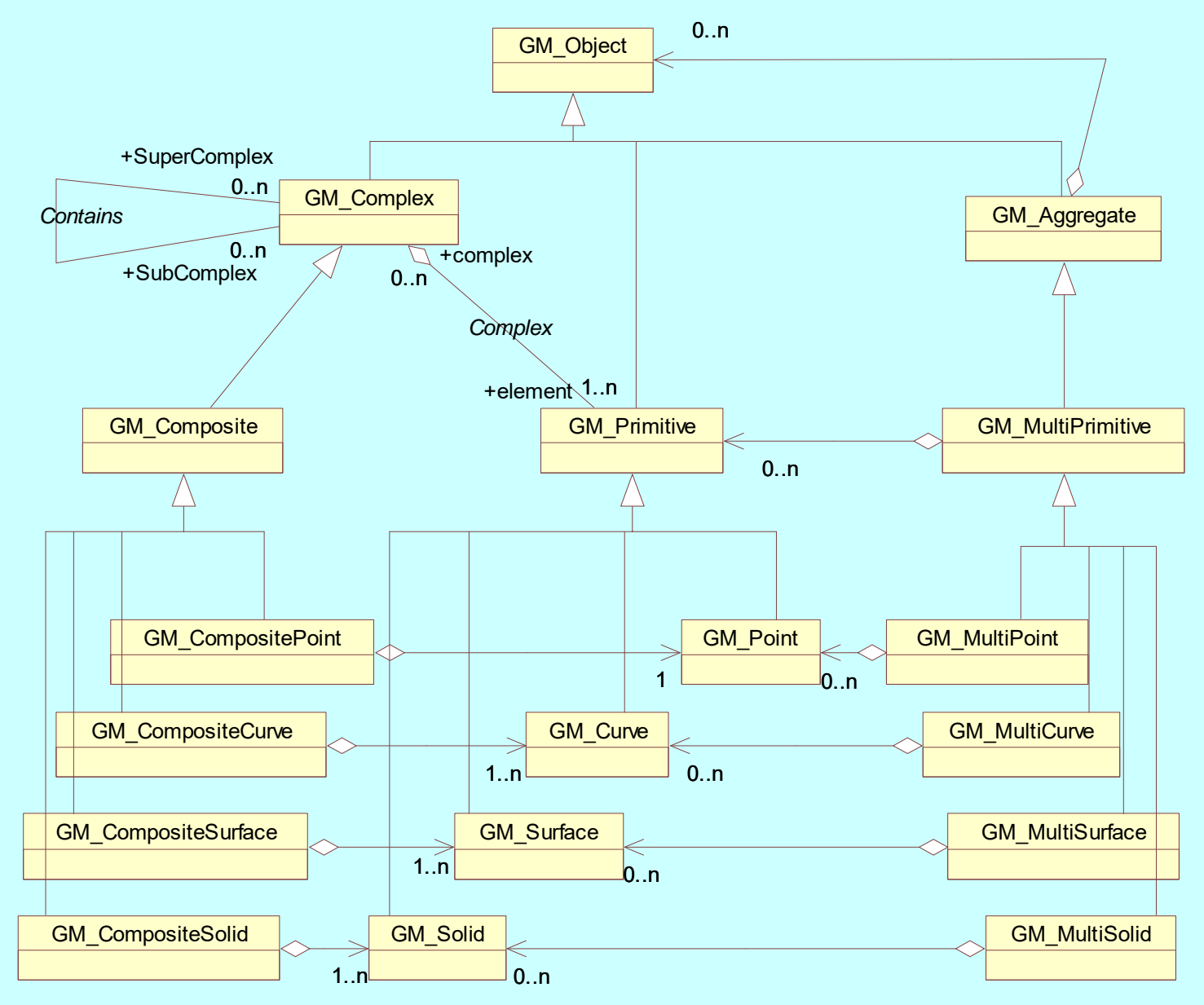

Figure 1. Main classes of the ISO Spatial Schema (standard ISO 19107).

An aggregate is a collection of geometric objects (GM_Objects) without any additional constraint. A complex object is a collection of simple (not self intersecting) and disjoint primitives in which the boundary of each primitive of the complex is a set of primitives in the same complex object (a complex object is closed). Complex objects are often built as sets of primitives representing a connected point-set, as for example in the case of the composite curve which is a connected set of curves having one start/end point pair; however no explicit associations exist among the primitives of a complex.

The topological relationships among complex objects can be defined using their object composition instead of their point-set representation, therefore avoiding the tolerance problems due to the computational geometry algorithms working on the coordinates of the point-set representation. In particular, the sharing of the geometry among complex objects (useful for topological constraints of SWP relationships) can be based on the Complex and the Contains associations of Figure 1.

The Complex association relates a complex object to its primitives and a primitive to the complex objects it belongs to. A shared geometry among complex objects can be therefore represented with a common set of primitives used in the composition of the complex objects; notice that, a shared geometry could be described also by using different primitives having the same point-set representation, but it implies that the topological relationships can be recognized on the point-set representation and cannot be further handled at the object composition level.

The Contains association establishes some sort of "structural subset relation" between two complex objects (seen as a whole); in fact; an instance of this association relates a contained complex object, in the role of SubComplex, to a container complex object in the role of 
SuperComplex and this assures that all the primitives of the subcomplex must belong to the set of the primitives of the supercomplex (total sharing of a complex object).

Notice that, if two or more complex objects are defined as SubComplexes of another common complex object, then their shared geometry is forced to be represented only once through the same set of primitives (the Complex association does not force it).

The structure of complex objects, the Complex and the Contains associations allow therefore the definition of topological constraints between complex objects both at the primitive level (e.g., each primitive of a transport network could be constrained to belong also to the complex object of some roads) and at the complex object level (e.g., the whole complex object of a road must belong to the complex object of a specific road network).

\section{The ISO Rules for Applications}

The Rules for Applications represent a guideline to define the conceptual schema of a spatial database, called by ISO application schema, in which parts of other standards (e.g., Spatial Schema) are used. The scope of this standard is to improve the semantic interoperability of data between different applications through a uniform behaviour of the designers.

This standard defines a General Feature Model (GFM) as an abstract conceptual model for geographic databases and then it describes standard rules to translate the GFM constructs into equivalent constructs of other conceptual models; in particular, it maps the GFM to the Unified Modelling Language (UML) data model. An UML based application schema will define class diagrams in order to describe data structures, in which the spatial data types of the Spatial Schema are used as domains of the spatial attributes, operations, relationships and OCL constraints.

The definition of a SWP relationship requires an association between the whole and the part classes and a topological constraint involving the spatial extents of the whole and the part classes. The constraint can be expressed through an OCL expression involving the classical point-set representation of the spatial extents, however, alternative methods are provided for the structural representation of spatial extents based on the GM_Complex type; each complex spatial extent is a set of primitive objects and therefore a topological constraint can be described using the Contains association or with an OCL expression working on the Complex association of the Spatial Schema. This paper will deeply analyze the structural approach which is suggested by ISO through the use of the Contains and the Complex associations for SWP relationships; notice that, the structural approach allows a better implementation of the sharing of spatial extents between whole and its parts and the result of the discussion remains valid also for the point-set representation.

\section{Modelling topological constraints of a SWP relationship through the Contains association}

The way in which SPW relationships can be modelled in the ISO TC211 approach is shown by the example described in Section 8.7.3.4 of Rules for Applications: "An overall transport network system is a collection of road networks defined on the basis of some administrative criteria, where each network consists of a set of roads".

This example of SPW relationship between a network and its roads is modelled in the standard as shown in Figure 2. 


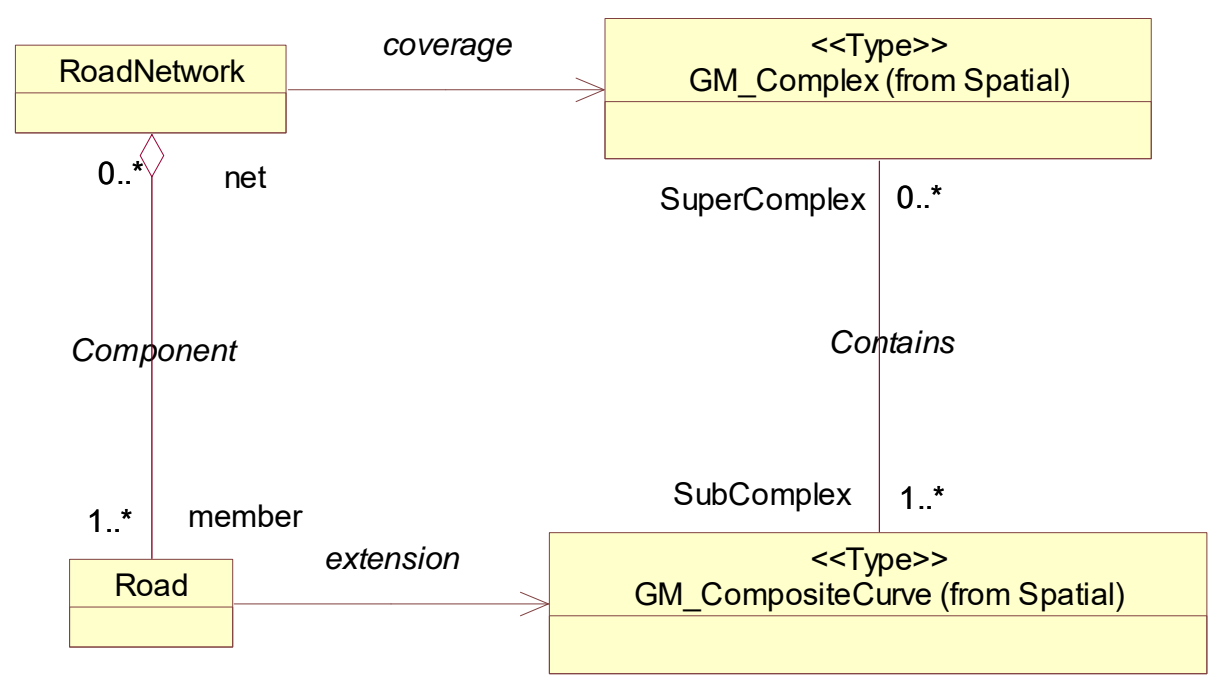

Figure 2. The application schema of Section 8.7.3.4 of Rules for Applications

The schema defines the RoadNetwork (the whole) and the Road (the parts) classes (basic rule 1, sec. 8.3.1 of Rule for Applications) and the association Component (basic rule 2, sec. 8.3.1 of Rule for Applications) to describe the administrative relationship between a network and its roads. The spatial representation of the geometric attribute of the two classes is expressed (rule 2, case 2, sec. 8.7.2 of Rule for Applications) through two associations (extension and coverage) between each class and the appropriated class of the Spatial Schema (GM_CompositeCurve for roads and GM_Complex for networks); the classes of the spatial schema are exposed in the schema in order to model the topological containment constraint between the geometric attributes of the classes through the use of the Contains association (rule 2, sec. 8.7.3.3 of Rule for Applications).

In the above example, the SWP relationship between Road and TransportNetwork is modelled separately at the semantic and geometric levels:

- the semantic relationship through one aggregation between the application classes;

- the topological constraint on the geometries through the Contains association.

This approach has the following drawbacks.

The optional membership of the Contains association. This association assures that its implicit constraint applies to the geometries which are members of the association and update operations cannot violate the constraint. However, the association does not impose any constraint on the geometries which are not members of the association. Let us consider a different situation in which the coverage of a network and the extension of its roads are not related in the Contains association; this satisfies the above application schema but it allows an independent definition and update of the geometry of roads and networks and therefore it shows that the Contains association alone cannot guarantee any topological constraint on data.

Notice that, this problem does not arise if a road belongs to one and only one network, since the cardinality of both the associations would impose to all the extensions of the roads to belong to the Contains association as expected. However, this does not apply in general.

The consistency of the associations between classes and geometries. The Contains association relates the geometries of the spatial attributes of the two classes, but it does not provide information about how the classes are related. Therefore, an additional explicit association between the two classes is necessary, called Component in the schema of Figure 2. However, the resulting schema admits an inconsistency between the content of the two associations (e.g., the extension of one road could be linked to a coverage of a network which is not its network in the Component association). 
The above problems can be removed attaching some additional OCL constraints to the schema in order to force topological constraints. As an example, let's consider the following OCL formulation of the topological constraint forcing the spatial extent of the network to be equal to the union of the spatial extent of the roads:

\section{context RoadNetwork}

inv:

((self.member $\rightarrow$ forAll(p: Road | p.extension.superComplex $\rightarrow$ includes(self.coverage) $))$ and

(self.coverage.element $=$ asSet(self.member.extension.element))

This constraint states that the primitives of the complex representing the coverage attribute of a network must be equivalent to the geometric union of the primitives of all the complexes representing the extension attribute of the roads which are subcomplexes of the complex of the network.

Notice that, the constraint imposes the mandatory participation in the Contains association, and moreover, it navigates over both the two associations in order to avoid the inconsistency between them.

\section{An ISO conformant systematic approach to model SWP relationships}

The above example has shown how a SWP relationship based on the equality topological constraint can be modelled in ISO by using OCL.

This section proposes an approach for defining ISO conformant modelling abstractions which hide the complexity of dealing with OCL constraints and it defines the minimal set of modelling abstractions for SWP relationships which cover all the SWP relationships of Table 1.

The proposed approach is based on :

- the definition of a schema template conforming to the ISO Spatial Schema and ISO Rules for Applications to cope with similar design situations;

- the addition of OCL formulas associated to the elements of the schema template for specifying the formal semantics of spatial constraints;

- the definition of a modelling abstraction which is a design oriented representation of the schema template together with a specific spatial constraint; a simple graphic representation is provided for each modelling abstraction in order to improve its use in database design.

The application of this approach to define modelling abstractions for SWP relationships is presented in the following subsections.

\subsection{The schema template}

The schema template for SWP relationships is a generalization of the ISO TC211 application schema shown for the transport network example, which allows shareability of parts and does not force existential dependency for whole and parts. It involves two classes (called Whole and Part) with spatial extents (gw and gp) modelled as associations to the GM_Complex class (or one of its subtypes), the Complex and the Contains associations of the Spatial Schema and a semantic association between Whole and Part classes. Figure 3 shows the template in which descriptive attributes have been omitted since they are not relevant. 


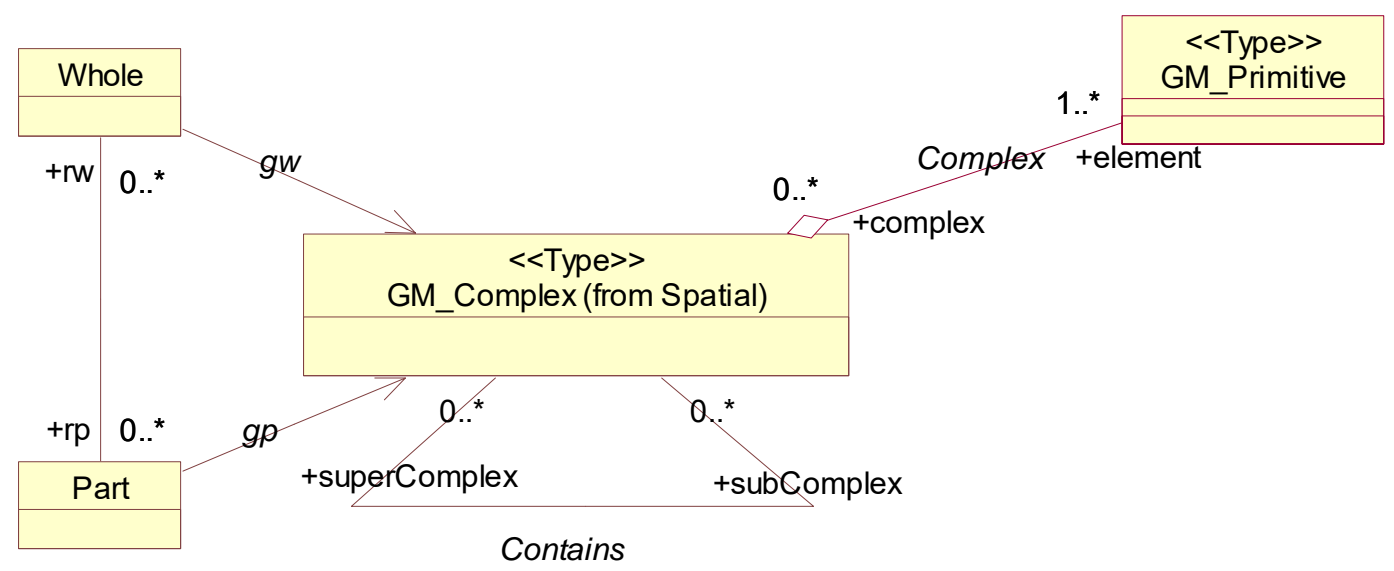

Figure 3. An ISO schema template for SWP relationships.

\subsection{Topological constraints for SWP relationships}

The classification of the constraints given in Section 2 require to enrich the ISO schema template with four topological constraints; the formal definition of these constraints, expressed using OCL v.1.4, is shown in Table 2.

\begin{tabular}{|c|c|}
\hline $\begin{array}{c}\text { Basic } \\
\text { topological } \\
\text { constraints }\end{array}$ & Formal definition in $O C L$ \\
\hline $\begin{array}{l}\text { Containment } \\
\text { constraint }\end{array}$ & $\begin{array}{l}\text { context Part } \\
\text { inv: (self.rw } \rightarrow \text { notEmpty) } \\
\quad \text { implies } \\
\quad(\text { self.rw } \rightarrow \text { forAll }(w: \text { Whole } \mid \text { self.gp.superComplex } \rightarrow \text { includes }(w . g w)))\end{array}$ \\
\hline $\begin{array}{l}\text { Covering } \\
\text { constraints }\end{array}$ & $\begin{array}{l}\text { context Whole } \\
\text { inv: (self.rp } \rightarrow \text { notEmpty) } \\
\quad \text { implies } \\
\quad \text { self.rp.gp.element -> includesAll(self.gw.element) }\end{array}$ \\
\hline $\begin{array}{l}\text { Equality } \\
\text { constraint }\end{array}$ & $\begin{array}{l}\text { context Whole } \\
\text { inv: } \\
\quad(\text { self.rp } \rightarrow \text { notEmpty) } \\
\quad \text { implies } \\
\quad((\text { self.rp } \rightarrow \text { forAll(p: Part } \mid \text { p.gp.superComplex } \rightarrow \text { includes(self.gw }))) \\
\quad \text { and } \\
\quad(\text { self.gw.element }=\text { asSet(self.rp.gp.element })))\end{array}$ \\
\hline $\begin{array}{l}\text { Equality } \\
\text { constraint } \\
\text { with } \\
\text { disjointness } \\
\text { parts }\end{array}$ & 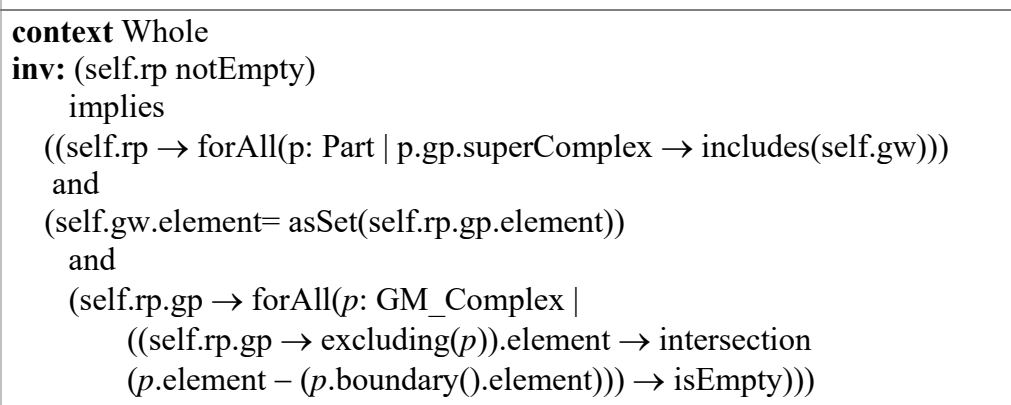 \\
\hline
\end{tabular}

Table 2: formal definition of the basic whole-part topological constraints. 
The "notEmpty" condition of the OCL constraints is required by the optional membership of the aggregation in the schema template; the condition assures that the topological constraint applies only to those instances of the Whole(Part) class involved in an instance of the aggregation. The containment constraint states that the geometry of each Whole of the constrained Part is a supercomplex of the geometry of the Part. The covering constraint assures that the geometry of a Whole is composed by a subset of the primitives of the geometry of its parts. The equality constraints assures that all the geometries of the Parts of a Whole are subcomplexes (and therefore contained in) of the geometry of the Whole and that the union of the geometry of the Parts correspond to the geometry of the Whole. The disjointness constraint is a Part-Part constraint that admits only overlapping of the parts boundaries.

The formulations of the semantics of the constraints of Table 2 take care also of the goal of having the constraints on the structure of the Contains and the Complex associations in order to guide their implementation.

The OCL constraints defined in the network example can be obtained by substituting the elements of the network application schema to those of the schema template in the above general constraint formulations.

Notice that, the topological constraints of Section 2 and all the SWP relationships proposed in the literature and shown in Table 1 are given in terms of intuitive descriptions and not formally defined, therefore the above formal definition of the topological constraints is a good basis for their interpretation in the ISO context, however a formal proof of their mapping into the above OCL constraints cannot be given.

\subsection{The modelling abstractions for SWP relationships}

Each SWP relationship described by the ISO schema template of Section 6.1 enriched with the related topological constraint of Table 2 can be collapsed in one modelling abstraction to be used as a design construct. Figure 4 shows the UML based graphical representation of the modelling abstractions of the following four SWP relationships: the "BelongsTo" relationship (containment constraint), the "CoveredBy" relationship (covering constraint), the "Partition" relationship (Equal constraint with part disjointness) and the "UnionOf" relationship (equal constraint).

Each modelling abstraction involves a Whole class and a Part class in which the spatial property is described using a spatial attribute (as foreseen in the ISO Rules for Applications) and the topological constraint is embedded in the semantics of a stereotype that specializes the association between the Whole and the Part classes.
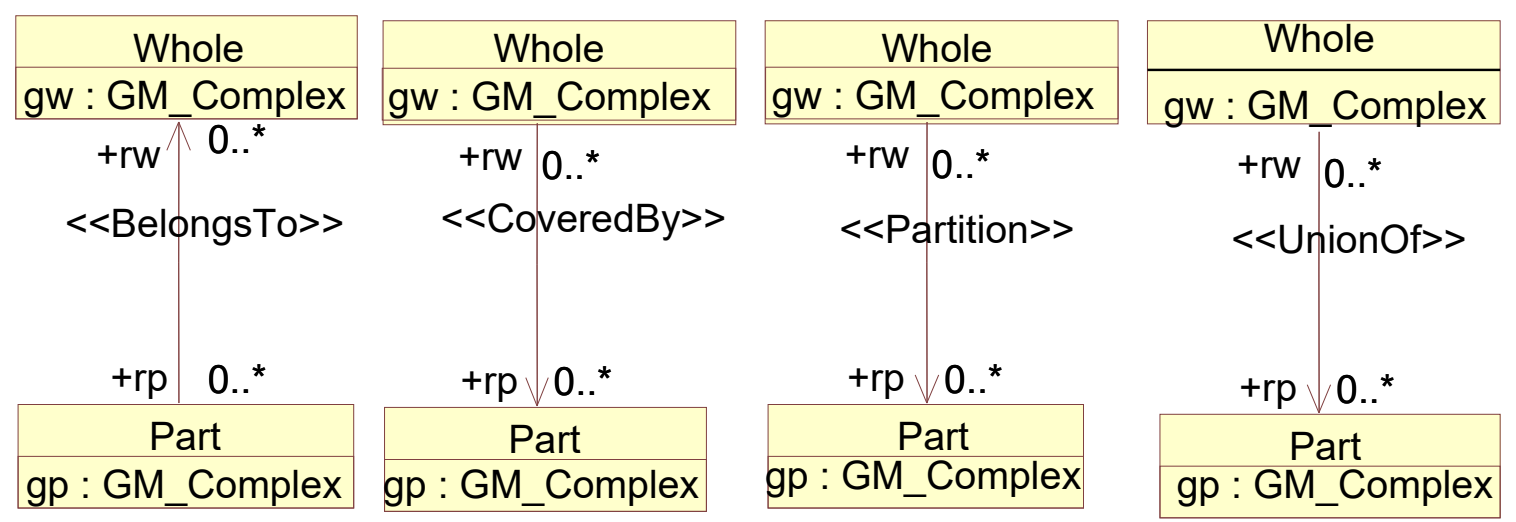

Figure 4. The graphic representation of the modelling abstractions for SWP relationships.

Notice that, the network example of section 5 could be therefore modelled using the "UnionOf" modelling abstraction between the RoadNetwork and the Road classes. 


\section{Conclusion}

The paper has shown that it is very difficult to specify SWP relationships through an application schema according to the ISO TC211 approach; in particular, non-trivial OCL formulas can be required for a correct schema specification. This leads to database schemas, which are not easy to read, understand and implement. It has been shown how powerful ISO compliant modelling abstractions with simple graphical representations can be defined as abstractions of ISO TC211 schemas enriched with OCL constraints. Moreover, specific modelling abstractions for SWP relationships have been defined and the SWP relationships proposed in literature have been described in terms of these abstractions.

The proposed approach improves the readability of the conceptual representation of data, but it also allows an improvement of the implementation, since the implementation of the Contains and Complex associations allows to store shared portions of geometry only once and to use them in several objects without replication. The topological data structures of some spatial DBMS are examples of a possible implementation of them.

Moreover, the equality constraint can lead to the notion of derivability since the spatial extent of the whole can be derived as the union of the spatial extent of the parts and therefore its storage can be avoided.

Future work could apply the proposed approach to consider all topological relationships in order to provide a minimal and complete set of modelling abstractions for spatial database design. A first attempt in this direction is given in [5]. The effectiveness of the proposed abstractions has been tested in the design of the conceptual schema of the Italian NSDI during the IntesaGIS project [32], where they cover all the types of spatial relationships specified in the schema.

\section{Bibliography}

[1] G. Abrantes, R. Carapuca, Explicit representation of data depend on topological relationships and control over data consistency, Proceedings of the 5th European Conference and Exhibition on GIS (EGIS/MARI), Paris, France, 1994, pp. 869-877.

[2] F. Barbier, B. Henderson-Sellers, A. Le Parc, J.M. Bruel, Formalization of the Whole-Part Relationship in the Unified Modeling Language, IEEE Trans. Software Eng. 29 (2003) 459-470.

[3] Y. Bédard, C. Caron, Z. Maamar, B. Moulin, D. Vallière, Adapting data models for the design of spatio-temporal databases, Computing environment and urban systems, 20 (1996) 19-41.

[4] Y. Bédard, Visual Modelling of Spatial Databases: towards spatial PVL and UML, GEOMATICA, 53 (1999) 169-186.

[5] A. Belussi, M. Negri, G. Pelagatti, GeoUML: an ISO TC 211 compatible data model for the conceptual design of geographic databases, Internal report N. 2004.21, Dipartimento di Elettronica e Informazione, Politecnico di Milano, Italy 2004.

[6] E. Bertino, G. Guerrini: Extending the ODMG Object Model with Composite Objects, Proceedings OOPSLA 1998, pp. 259-270.

[7] K.A.V. Borges, A.H.F. Laender, C.A. Davis, Spatial Data Integrity Constraints in Object Oriented Geographic Data Modeling, Proceedings of the 7th ACM-GIS 1999, pp.1-6.

[8] K.A.V. Borges, C.A. Davis, A.H.F. Laender, OMT-G: An Object Oriented Data Model for Geographic Applications, GeoInformatica, 5 (2001) 221-260.

[9] J. Brodeur, Y. Bédard, M.J. Proulx, Modelling geospatial application databases using UML based repositories aligned with international standards in geomatics, Proceedings of the 8th ACM-GIS 2000, pp. 39-46.

[10] A.F. Christensen, N. Tryfona, C.S. Jensen, Requirements and Research Issues in Geographic Data modeling, Proceedings ACM International Symposium on Advances in Geographic Information Systems, Atlanta, GA, USA, 2001, pp. 2-8.

[11] S. Cockcroft, The design and Implementation of as repository for the management of spatial data integrity constraints, GeoInformatica, 8 (2004), 49-69.

[12] J. Filho, C. Iochpe, Specifying Analysis Patterns for Geographic Databases on the basis of a Conceptual Framework, Proceeding of the 7th ACM GIS, 1999, pp.7-13. 
[13] S. Gordillo, F. Balaguer, C. Mostaccio, F.D. Neves, Developing GIS applications with objects. A design pattern approach, GeoInformatica, 3 (1999) 7-32.

[14] T. Hadzilacos, N. Tryfona, An Extended Entity-Relationship Model for Geographic Applications, SIGMOD Record 26 (1997) 24-29.

[15] M. Halper, J. Geller, Y. Perl, An OODB Part-Whole Model: Semantics, Notation and Implementation, Data Knowl. Eng. 27 (1998) 59-95.

[16] http://www.intesagis.it/specifiche/Doc_wg01/1n1007_4.pdf

[17] ISO/TC 211 Geographic information/Geomatics, 19107 Geographic information - Spatial schema, text for FDIS, N. 1324 (2002)..

[18] ISO/TC 211, Geographic information/Geomatics, 19109, Geographic Information - Rules for application schema, text for FDIS, N. 1538 (2003).

[19] ISO/TC 211, Geographic information/Geomatics, 19115, Geographic Information - Simple feature access, text for DIS, N. 1003 (2000).

[20] M.A. Ketabchi, V. Berzins, Mathematical Model of Composite Objects and Its Application for Organizing Engineering Databases, IEEE Trans. Software Eng. 14 (1988) 71-84.

[21] W. Kim, J. Banerjee, H.T. Chou, J.F. Garza, D. Woelk, Composite object support in an objectoriented database system, Conference on Object Oriented Programming Systems Languages and Applications Orlando, Florida, United States, 1987 pp. 118-125,

[22] G.Kösters, B.Pagel, H.W. Six, GIS-application development with GeoOOA, International Journal of GIS, 11 (1997), 307-335.

[23] J.L. Oliveira, F. Pires, C.M.B. Medeiros, An environment for modelling and design of geographic applications, GeoInformatica, 1 (1997) 29-58.

[24] A.L. Opdahl, B. Henderson-Sellers, F. Barbier, Ontological analysis of whole-part relationships in OO-models, Information \& Software Technology 43 (2001) 387-399.

[25] Open GIS Consortium, Inc., OpenGIS Simple Feature Specification for SQL, Revision 1.1, N. 99-049, 1999.

[26] C. Parent, S. Spaccapietra, E. Zimanyi, P. Donini, C. Plazanet, C. Vangenot, Modeling spatial data in the MADS conceptual model, Proc. of the 8th Int. Symposium on Spatial Data Handling, Vancouver,Canada, 1998, pp. 138-150.

[27] R. Price, N. Tryfona, C.S. Jensen, Modeling Part-Whole Relationships for Spatial data, Proceedings of the 8th ACM GIS, Washington D.C., USA, 2000, pp. 1-8.

[28] R. Price, N. Tryfona, C.S. Jensen, Modelling Topological Constraints in Spatial Part-Whole Relationships, ER 2001, LNCS N. 2224, pp. 27-40.

[29] J. Rumbaugh, I. Jacobson, G. Booch, The Unified Modeling Language Reference Manual, Boston: Addison-Wesley, 1999.

[30] S.Shekhar, M.Coyle, B. Goyal, D.Liu, S.Sarkar, Data models in geographic information systems, Communications of the ACM, 40 (1997) 103-111.

[31] V.C. Storey: Understanding Semantic Relationships, VLDB J. 2 (1993) 455-488.

[32] M.F. Worboys, H.M. Hearnshaw, D.J. Maguire, Object oriented modelling for spatial databases, Int. journal of GIS 4 (1990) 369- 383. 